Association for Information Systems AIS Electronic Library (AISeL)

December 2004

\title{
An Empirical Examination of the Effects of Web Personalization at Different Stages of Decision- Making
}

Shuk Ho

Hong Kong University of Science and Technology

Kar-Yan Tam

Hong Kong University of Science and Technology

Follow this and additional works at: http://aisel.aisnet.org/amcis2004

\section{Recommended Citation}

Ho, Shuk and Tam, Kar-Yan, "An Empirical Examination of the Effects of Web Personalization at Different Stages of Decision-Making" (2004). AMCIS 2004 Proceedings. 425.

http://aisel.aisnet.org/amcis2004/425

This material is brought to you by the Americas Conference on Information Systems (AMCIS) at AIS Electronic Library (AISeL). It has been accepted for inclusion in AMCIS 2004 Proceedings by an authorized administrator of AIS Electronic Library (AISeL). For more information, please contact elibrary@aisnet.org. 


\title{
An Empirical Examination of the Effects of Web Personalization at Different Stages of Decision-Making
}

\author{
Shuk Ying Ho \\ Department of Information and Systems \\ Management \\ Hong Kong University of Science and Technology \\ Clear Water Bay, Hong Kong \\ susanna@,ust.hk
}

\author{
Kar Yan Tam \\ Department of Information and Systems \\ Management \\ Hong Kong University of Science and Technology \\ Clear Water Bay, Hong Kong \\ kytam@ust.hk
}

\begin{abstract}
Personalization agents are incorporated in many websites to tailor content and interfaces for individual users. But in contrast to the proliferation of personalized web services worldwide, empirical research studying the effects of web personalization is scant. How does exposure to personalized offers affect subsequent product consideration and choice outcome? Drawing on the literature in HCI and consumer behavior, this research examines the effects of web personalization on users' information processing and expectations through different decision-making stages. A study using a personalized ring-tone download website was conducted. Our findings provide empirical evidence of the effects of web personalization. In particular, when consumers are forming their consideration sets, the agents have the capacity to help them discover new products and generate demand for unfamiliar products. Once the users have arrived at their choice, however, the persuasive effects from the personalization agent diminish. These results establish that adaptive role of personalization agents at different stages of decision-making.
\end{abstract}

\section{Keywords}

Web Personalization, Intelligent Agent, Awareness Set, Consideration Set, Choice, Decision-Making.

\section{SECTION 1: INTRODUCTION}

Personalized information services have become ubiquitous in many business-to-customer applications. Investment in content personalization is expected to grow to an estimated US\$6 billion by 2004 (Ledford, 2002). It is one of the fastest-growing areas of IT (Ramakrishnan, 2000). While most IS work address personalized services in general, our work focuses on web personalization, which refers to the process of adapting web content and web layout with the dual objectives of serving users' needs as well as maximizing business opportunities. The goal is to deliver the right content to the right person in the right format at the right time.

Personalization agents empower online merchants to exert control and manipulate web content at a very fine level previously not possible. Online merchants are able to manipulate presentation timing and layout of a page to adapt to the user's needs and at the same time to maximize their business opportunities. One of the most recognized successful examples is Amazon.com, which has constructed a customer-centric online store and provides one-to-one services to over 20 million customers. Collaborative-filtering technology is used to match customer profiles with those of like-minded customers who share similar preferences and interests. Book recommendations congruent with returning customers' past purchases are then offered. This reduces product search costs and offers a unique experience to Amazon's customers.

\subsection{Prior Information Systems (IS) Research on Web Personalization}

With the proliferation of personalized web services worldwide, personalization is drawing increasing attention among researchers. Previous work related to personalization falls into three main streams. The first stream includes studies on the applications of personalization technology. Personalization agents are found to be useful in different domains such as information dissemination (Foltz and Dumais, 1992; Loeb, 1992; Light and Maybury, 2002), search engines (Manber, Patel 
and Robison, 2000; Pitkow, Schütze, Cass, Cooley, Turnbull, Edmonds, Adar and Breuel, 2002), medicine (Bental and Cawsey, 2002) and online retailing (Maes, Guttman and Moukas, 1999). These studies highlight new applications of personalization technology and their commercial potential. The second stream focuses on privacy issues related to personalization (Kobsa, 2002; Stewart and Segars, 2002; Volokh, 2000). Users face a dilemma: while they demand more customized services on one hand, they are increasingly concerned about privacy infringements and how their information is being used by online merchants on the other hand. These studies address issues centered around this dilemma. The third stream focuses on enabling technologies in mining the enormous amount of customer transactions and deriving efficient rules to generate personalized content (Dasgupta and Melliar-Smith 2003; Eirinaki and Vazirgiannis 2003; Perkowitz and Etzioni 2000; Ramakrishnan 2000; Shahabi and Banaei-Kashani 2003). Work in this area concentrates on computational procedures to mine transactions and personal profiles. While these studies focus on various aspects of personalization applications, there has been very little work that considers the theoretical basis for understanding the relationships between personalization and user behavior.

\subsection{Motivation and Research Questions}

Recently, Murthi and Sarkar (2003) suggested more research directions to study online personalization in the context of management science. They noted that one of the most important questions to practitioners using web personalization systems is Which items should be offered to influence individual customers' consideration sets? (Murthi and Sarkar, 2003, pp.1357). In the current work, we focus on both the personalization content and the presentation time. In particular, we study the robustness of personalization recommendations under different decision-making stages of a customer. We examine whether exposing a customer to personalized recommendations at different decision-making stages have impact on the likelihood of their subsequent inclusion in the customer's consideration set and his choice outcome. We address the following research questions:

1. Whether web personalization increases the likelihood of the recommended item to be included in the consideration set?

2. Whether web personalization increases the likelihood of the recommended item to be selected as the final choice?

Three reasons guide our focus. First, inclusion of a product in a consideration set is often a necessary condition for choice (Howard and Sheth, 1969; Howard, 1989). Determining whether or not, and under what conditions, personalized exposure affects the formation of consideration sets would help us understand the effects of information processing on web advertising effectiveness. Second, from a theoretical perspective, this research provides a better understanding of the role of a personalization agent in enhancing various aspects of consumer decision-making. Our analysis helps us to understand when a consumer's judgment is sensitive to the personalized stimuli. Third, an understanding of whether or not personalized decision aids influence consumer decision-making across users who are at different decision-making stages will generate useful insights to online businesses interested in deploying personalization agents.

\section{SECTION 2: THEORETICAL PERSPECTIVES AND HYPOTHESIS DEVELOPMENT}

When consumers perform shopping tasks online, they employ a multi-stage mechanism to come up with their final choice (see Figure 1).

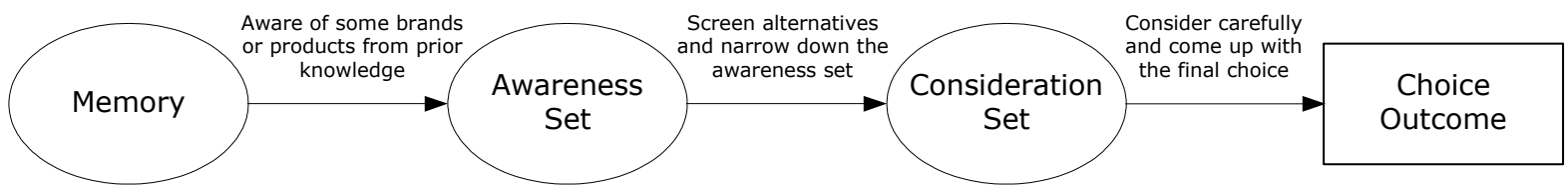

Figure 1. Information Processing Model

\subsection{Awareness Set}

Web content can be conceptualized as a mix of stimuli that take the form of text, images, audio, animations or video. In the course of interacting with a web page, these stimuli divert attention, reallocate cognitive resources, and evoke affective 
responses and behaviors. But with limited cognitive resources, consumers can only be aware of a few brands or products. These items - a subset of the stimulated items - are included in their awareness set.

A number of variables influence the content of the awareness set. These include attention, comprehension, expertise, processing capacity, processing effort and opportunity to process. Generally, brands related to one self or past episodic experience are more accessible from memory (Burnkrant and Unnava, 1989; Burnkrant and Unnava, 1995). Frequently (or recently) encountered brands are more accessible from memory than brands infrequently encountered (or brands encountered long ago). Also, external stimuli can serve as cues to help recalling information from memory. These stimuli may include, for example, the stimuli of text, images, etc. that a user encounters while shopping online.

\section{$2.2 \quad$ Consideration Set}

Consumers screen the alternatives to narrow down their awareness set (Desai and Hoyer, 2000; Punj and Brookes, 2001) to form the consideration set. This set consists of products that they would consider carefully for decision-making (Roberts and Lattin, 1991). In general, accessible brands from awareness set are likely to be included in the consideration set.

Online merchants would be interested to know whether a web personalization agent can influence consumers' consideration set when personalized recommendations are presented at the early decision-making stage (e.g. T1 in Figure 2)?

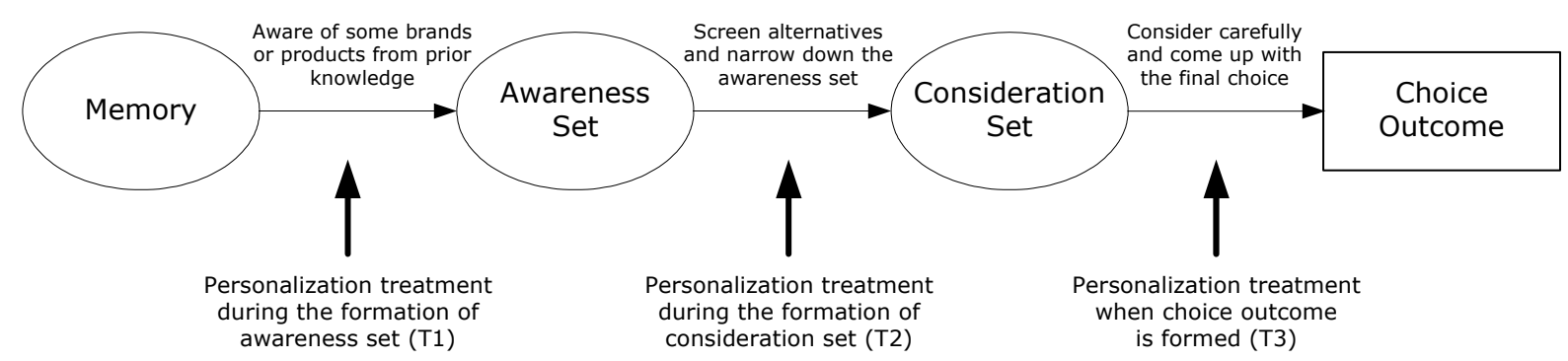

Figure 2. Presentation Timing of Personalized Recommendations

Usually, a personalization agent uses eye-catching symbols to make the recommendations salient. Further, the personalized recommendations often match personal preferences or the past episodic experience of the consumers. Both effects facilitate information about these recommendations to be retrieved and included in the awareness set. In summary, a stimulus at T1 may ultimately influence the consideration set, i.e. through the effect of the awareness set directly on the consideration set. Personalized products matching one's personal preferences may cause the consumer to retrieve that product name from memory. This may be particularly important example of how stimuli can effect the consideration set via retrieval, as product information retrieval is known to exert strong effects on the formation of a consideration set (Nedungadi, 1990). Thus, we propose the following:

\section{H1 The likelihood of a personalized item entering the consideration set is higher if the personalized item} matches one's personal preferences.

At the same time, it is also interesting to know whether a web personalization agent can influence consumers' consideration set when personalized recommendations are presented at the later decision-making stages (e.g. T2 in Figure 2)? Past research provides overwhelming evidence that consumers have the motivation to keep the consideration set small, because a large consideration set increases demand on more cognitive resources associated with the extra effort required to evaluate the attractiveness of alternatives (Huffman and Kahn, 1998; Shugan, 1980). Thus, we postulate that by the time the consideration set is being formed, it may be "too late" to exert a strong influence on the decision-making process:

H2 The likelihood of a personalized item entering the consideration set is lower if the user has already formed his consideration set. 
Online merchants would also be interested in knowing the effect of a personalized recommendation at the very late stage of the consumer's decision-making process? Prior research suggests that comparisons among available alternatives are much easier when alternatives are similar (Johnson, 1984; Johnson 1986; Levin and Jasper, 1995; Tversky, 1977). Thus, consumers are motivated to retain alternatives that have a considerable amount of overlapping features.

H3 After a user has formed his consideration set, a personalized item that has stronger overlapping with items already inside the consideration set is more likely to be included in the consideration set than one that has less overlapping.

\subsection{Choice Outcome}

After the formation of consideration set, consumers go through a process of eliminating alternatives from the consideration set to form their choice outcome. That is, consumers evaluate each alternative in the consideration set to select the best alternative(s).

How does a web personalization agent influence consumers' choice outcome? This depends upon when the personalization is presented. The first scenario is that the personalization is presented at T1 in Figure 2. In this case, the personalization will have influenced formation of the consideration set. Since decision costs (the costs involved in selecting one brand from the set of considered brands) are dependent on evaluation costs (the cost involved in determining whether or not to include a brand in the consideration set), personalized items matching one's personal preferences should be more likely to be chosen simply because they are more likely to be included in the consideration set (Hauser and Wernerfelt, 1990). Hence, personalized items matching one's personal preferences are more likely to be included in the choice outcome by first influencing the composition of the consideration sets.

H4 The likelihood of a personalized item being accepted is higher if the personalized item matches the user's personal preferences.

Is a web personalization agent effective in influencing consumers' choice outcome when personalized recommendations are presented at the late decision-making stage (e.g. T2 in Figure 2)? Following the arguments in H3, we postulate that the chance of personalized recommendations getting into the consideration set drops, and this leads to a smaller chance of a late personalized item to be the choice outcome.

If personalized recommendations are presented after the choice outcome is formed (e.g. T3 in Figure 2), can consumers still be persuaded to change his choice? Generally, a large number of choices increase demand on a user's cognitive resources associated with the extra effort required to evaluate the attractiveness of alternatives (Huffman and Kahn, 1998; Shugan, 1980). Also, changing choice outcome after its formation might confuse consumers, leading to weaker preferences and lower choice probability (Dhar, 1997; Greenleaf and Lehmann, 1995; Iyengar and Lepper, 2000). Consequently, the persuasiveness of web personalization agent is expected to be diminished.

\section{H5 The likelihood of a personalized item being accepted is lower if the user has formed his consideration} set.

We conducted a study using a personalized ring-tone recommendation website. Ring-tones are MIDI files, melodies or part of a music file used in a cellular phone ringer. Data were collected to examine and empirically demonstrate the above hypotheses.

\section{SECTION 3: PERSONALIZED RING-TONE DOWNLOAD STUDY}

\subsection{Participants}

We cooperated with the largest mobile data services content provider in Hong Kong to conduct this study. Emails were sent to its members for recruitment. All of them were ring-tone users. As a token of appreciation, the respondents earned a free ring-tone for their own cellular phones and a chance to join a lucky draw for a special gift. To make the navigation environment more natural, people could perform their tasks at anytime and from any place within a six-week period. 


\section{$3.2 \quad$ Procedures}

All participants received an email about the availability of a personalization service which was to be incorporated in the ringtone download website. This service would have a customized layout, and users would have some ring-tones recommended based on their ring-tone download history and singer preferences. The members were asked to go to the website and fill in a questionnaire to evaluate its performance. They could go to the website by clicking on a link embedded in the email at any time and from any place, but they could evaluate the web personalization agent only once.

The study was divided into three parts. First, the participants were asked to fill in a questionnaire about their demographic information and ring-tone download habits. We motivated them to fill in the questionnaire carefully by saying that personalized offers would be generated based on their answers.

Second, we asked the participants to indicate their preferences for rhythms and singers. They chose and ranked their three most favorite singers from a list of 18 singers. Information from the Hong Kong Music Billboard allowed us to determine which songs were popular the latest albums.

Finally, all respondents entered a webpage that had 12 ring-tone choices. There were two buttons next to each ring-tone. One button was the "LISTEN" button, whereas the other was the "DOWNLOAD" button. The respondents could listen any number of ring-tones and download exactly one ring-tone free of charge. When they confirmed to download a ring-tone, the selected ring-tone was sent to the participant's cellular phone via short messages service. All click streams were recorded.

A pretest with 56 subjects was used to validate the questionnaire and test the ring-tone download system performance. Participants could complete the whole process in 25 minutes. These 56 subjects agreed that the navigation process was smooth. They also noted that the blinking personalized banner did not interrupt the selection task.

\subsection{Design and Manipulation}

We first studied the transaction log from the mobile service provider. This log contained the real ring-tone purchases of 7,858 distinct users. There were 66,795 transactions dated from August 2002 to November 2003. These users downloaded ringtones from 175 distinct singers. We chose the top 18 singers, who accounted for 32,869 (by 6,474 distinct users) out of 66,795 download transactions.

We then formed a pool of 72 ring-tones from 18 singers (4 ring-tones per singer). Most singers had two ring-tones in fast rhythms, and the other two in slow rhythms. The ring-tones in the same rhythm category were assigned a recommendation priority based on the information from the Hong Kong Music Billboard. All participants received a list of 12 ring-tones. Six ring-tones were presented under the column Personalized Offers, whereas the other six were presented under the column Other Offers.

\subsection{Manipulations of Independent Variables}

Our work was made up of two parts. In the first part, a 2 (personalized recommendation timing) $\times 2$ (matching personal preferences) between-subject design was used. In the second part, a 2 (personalized recommendation timing) $\times 2$ (feature overlapping) between-subject design was employed.

\section{(a) Personalized Recommendations Presentation Timing}

In Part 1, there were two levels of presentation timing manipulations: at the formation of awareness set (T1) and at the formation of consideration set (T2). Two groups received their personalized recommendations once they logged on to the ring-tone download interface. At that moment, participants had no ideas about what ring tones were available. Thus, personalized recommendations were presented early at the formation of awareness set.

The other two groups in Part 1 received a pop-up message and participants in these groups were asked if they wanted to view their personalized recommendations after they had listened two ring-tones. At that moment, participants had screened all choices and considered two items seriously. Thus, participants in these two groups were manipulated to have their personalized recommendations at the time of consideration set formation. They could choose not to view the personalized offers. 
In Part 2, there were two levels of presentation timing manipulation: at the formation of consideration set (T2) and after the formation of choice outcome (T3). Two groups in Part 2 had the same timing manipulation as the last two groups in Part 1. That is, participants received a pop-up message and were asked if they wanted to view their personalized recommendations after they had listened two ring-tones.

The other two groups in Part 2 were manipulated differently. Participants in these groups encountered a pop-up message after they clicked on the "DOWNLOAD" button. They were asked if they wanted to view their personalized recommendations. That is, participants in these groups received personalized recommendations only after they had formed their choice outcome.

In the following analysis, we focused on both the composition of consideration sets and the choice outcome if personalized recommendations were presented at $\mathrm{T} 1$ or $\mathrm{T} 2$. If personalized recommendations were presented at $\mathrm{T} 3$, we only focused on the choice outcome, but not the composition of the consideration set (see Table 1).

\begin{tabular}{|l|l|l|l|}
\hline Decision Stage & Manipulation & $\begin{array}{l}\text { Composition of } \\
\text { Consideration Set }\end{array}$ & Choice Outcome \\
\hline $\begin{array}{l}\text { At the Formation of } \\
\text { Awareness Set (T1) }\end{array}$ & Once logon & $\checkmark$ & $\checkmark$ \\
\hline $\begin{array}{l}\text { At the Formation of } \\
\text { Consideration Set (T2) }\end{array}$ & After 2 ring-tone listens & $\checkmark$ & $\checkmark$ \\
\hline $\begin{array}{l}\text { After the Formation of } \\
\text { Choice Outcome (T3) }\end{array}$ & After download choice made & $\times$ & $\checkmark$ \\
\hline
\end{tabular}

Table 1. Measurement of Dependent Variables

\section{(b) Matching Personal Preferences}

There were two levels of manipulations: matching personal preferences and not matching personal preferences. Recommendations were said to be matching a participant's personal preferences when personalized recommendations belonged to his favorite singers and rhythm. Recommendations were said to be not matching a participant's personal preferences when personalized recommendations were extracted from the ring-tone pool randomly.

\section{(c) Feature Overlapping}

There were two levels of manipulations: strong feature overlapping and weak feature overlapping. To estimate the strength of feature overlapping between personalized recommendations and items in the consideration sets, we first classified the singers into groups. We analyzed the transaction log again to measure similarity among singers. For each of the 6,474 distinct users, we counted the number of downloads per singer for the 18 singers and then employed principal component analysis. Two groups of singers were formed, and each group contained nine singers. If two ring-tones belong to singers in same group, then these ring-tones were said to have strong feature overlapping. If they belong to singer in different group, then these ringtones were said to have weak feature overlapping.

\subsection{Dependent Variables}

\section{(a) Composition of Consideration Set}

Before downloading the ring-tones, participants could press the "LISTEN" button to hear the ring-tone first. They could listen any number of ring-tones. The composition of a participant's consideration set was recorded from the sequence of ringtone that the participant heard. In our data analysis, we focused on whether personalized ring-tones being elaborated. Thus, the dependent variable was operationalized to the listened ring-tones on the personalized recommendation list.

\section{(b) Final Choice}

The participants pressed the "DOWNLOAD" button when they made a choice. The nature of the final choice (personalized / non-personalized) was recorded. The dependent variable was the acceptance of personalized recommendations. It was a binary variable ( $1=$ download a personalized ring-tone; $0=$ download a non-personalized ring-tone). 


\subsection{Results}

Part 1: Personalized Recommendation Timing $\times$ Matching Personal Preferences

There were 408 participants (173 females and 235 males), and their average age was 24.72 years old. They were randomly assigned to one of the treatment groups (see Table 2).

\begin{tabular}{|l|l|l|l|}
\hline Group & Presentation Timing & Matching Preference? & No. of Responses \\
\hline 1 & \multirow{2}{*}{ Once logon } & Yes & 99 \\
\cline { 3 - 4 } 2 & & No & 101 \\
\hline 3 & \multirow{2}{*}{ After 2 ring-tone listens } & Yes & 104 \\
\cline { 3 - 4 } & & No & 104 \\
\hline 4 & & 408 \\
\hline \multicolumn{2}{|l|}{ Total: }
\end{tabular}

Table 2. Treatment in Part 1

\section{(a) Composition of Consideration Set}

Two-way ANOVA was conducted, with personalized recommendation timing and matching personal preferences as the explanatory variables. The dependent variable was the number of listened personalized ring-tones. Results show that participants were willing to spend efforts on considering recommendations matching their personal preferences (mean=3.72), but not on considering recommendations not matching their personal preferences (mean=3.08). Hence, H1 is supported $(F(1,404)=272.12, p<0.01)$.

If personalized recommendations are presented at the early decision-making stage, they have a high chance to be considered. Early recommendations (mean=4.25) were considered more often than late recommendations (mean=2.59), supporting H2 $(F(1,404)=40.07, p<0.01)$.

\section{(b) Final Choice}

A logistic regression was conducted with the same explanatory variables in the previous analysis. The dependent variable was the acceptance of a personalized ring-tone. Results show that ring-tones matching a participant's personal preferences (mean $=0.48)$ were downloaded more often than those not matching his personal preferences (mean=0.35), supporting H4 $\left(\chi^{2}(1)=6.46, p<0.05\right)$.

Also, personalized recommendations presented at the early decision-making stage (mean=0.52) were downloaded more often than personalized recommendations presented at the late decision-making stage (mean=0.32). Hence, H5 is supported $\left(\chi^{2}(1)=15.63, p<0.01\right)$.

\section{Part 2: Personalized Recommendation Timing $\times$ Feature Overlapping}

There were 412 participants (173 females and 239 males), and their average age was 25.02 years old. They were randomly assigned to a treatment group (see Table 3).

\begin{tabular}{|l|l|l|l|l|}
\hline Group & Presentation Timing & Feature Overlapping? & No. of Responses & No. of Usable Responses \\
\hline 1 & \multirow{2}{*}{ After 2 ring-tone listens } & Strong & 109 & 94 \\
\cline { 3 - 5 } & & Weak & 99 & 85 \\
\hline 3 & \multirow{2}{*}{$\begin{array}{l}\text { After download choice } \\
\text { made }\end{array}$} & Strong & 101 & 90 \\
\cline { 3 - 5 } & Weak & 103 & 80 \\
\hline \multicolumn{2}{|l|}{ Total: } & & 412 & 349 \\
\hline
\end{tabular}

Table 3. Treatment in Part 2 
Among 412 participants, $63(=15.29 \%)$ participants selected their top three favorite singers who belonged to different singer groups. We could not manipulate the factor, feature overlapping, for these participants; hence we dropped these data points. Our analysis focused on 349 participants.

\section{(a) Composition of Consideration Set}

We focused on Groups 1 and 2 only, because only participants in these groups received personalization treatment before the complete formation of consideration set. One-way ANOVA was conducted, with feature overlapping as the explanatory variables. Results show that participants were more willing to consider recommendations that were similar to the items in their consideration sets $(m e a n=3.02)$ than that were different from the considered items (mean=2.61). H3 is supported $(F(1,177)=7.27, p<0.01)$.

\section{(b) Final Choice}

A logistic regression was conducted with personalized recommendation timing and feature overlapping as the same explanatory variables. Results of this analysis show that personalized recommendations that were presented at the early decision stage could bias participants' decision. If personalized recommendations are presented when participants listen the ring-tones, the chance of a personalized item to be downloaded is 0.47 . If the recommendations are presented when participants make a choice, the choice is dropped to 0.37. Again, consistent with Part 1, H5 is supported $\left(\chi^{2}(1)=7.57, p<0.01\right)$.

\section{SECTION 4: DISCUSSION}

In contrast to the widespread adoption of personalization software and the strong advocacy by management gurus (Porter, 2001) on the use of personalization services as a differentiating strategy, little has been done to assess the effectiveness of web personalization. The purpose of this study was to understand the influence of different timing and content manipulations by personalization agents. Our work empirically assesses the effects of web personalization. We attempt to bridge this gap and provide an extensible theoretical framework to investigate issues related to personalized IT services. Major contributions are summarized below.

First, personalization is to provide the right content in the right format to a person at a right time. Yet, recent research on personalization has mainly focused on content personalization (Loeb, 1992; Pitkow et al., 2002) and only a few articles have looked at the web interface personalization (Billsus, Brunk, Evans, Gladish and Pazzani, 2002; Manber et al., 2000). Researchers overlook the importance of timing of when to present the personalized offers. The current work represents a pioneering effort to study the impacts of web personalization on user behavior and judgment, such that the personalized recommendations are given at different decision-making stages.

Second, the current work makes reference to consideration set theory to describe how users react in the face of a personalization agent that presents recommendations at different consumer decision-making stages. Our empirical evidences show that the persuasive effects from personalization agents are the strongest at the earliest decision-making stage. With more knowledge about the user navigation modes from their click streams (Moe, 2003), personalization agents should be able to determine the users' decision-making stage and thus adapt accordingly. For instance, when consumers have formed their choice, the personalization agent could act as a price-comparison agent, rather than offer recommendations.

Third, practitioners will also be interested in the implications of this research for the design of personalized websites. Part 1 in our study demonstrates the importance of recommendation content quality. Consumers like to explore information matching their preferences. Part 2 in our study demonstrates that when consumers have formed their consideration set, they tend to continue exploring similar alternatives. Both imply that detecting navigation goal and consumers' preferences or from their click streams are crucial.

\section{SECTION 5: CONCLUSION}

We have investigated the effects of web personalization in terms of the user's decision-making stages. This study represents a first step toward understanding how web personalization impacts on choice consideration and choice outcome. It also sheds light on the significance of personalization to online merchants in offering unique experiences to its users. Generally, users are more willing to explore the personalized content further at the early stage of decision-making, and this results in high level of information exploratory behavior. Once the users have arrived at their choices, however, the persuasive effects from 
the personalization agent diminish. These results establish that personalization agents should adapt to the consumers' different stages of decision-making.

There are a variety of ways to extend our work. First, in this study, the subjects were invited to an artificial website, so that we could ensure that this was their first time to experience the power of personalization at this site. What would happen if the visitors returned to the site? Would they rely on the personalized service in the long run? An actual field study might contribute much to this area of research. Second, while our work focuses on how personalization influences the formation of a stimulus-based consideration set, personalized advertisements can also implant a message in the memory for memory-based decision-making in the coming visits. It might be possible to perform laboratory studies to understand the impacts of personalization in a memory-based decision-making environment.

\section{REFERENCES}

1. Bental, D., and Cawsey, A. (2002) The Adaptive Web: Personalized and Adaptive Systems for Medical Consumer Applications, Communications of the ACM, 45, 5, 62-63.

2. Billsus, D., Brunk, C.A., Evans, C., Gladish, B. and Pazzani, M. (2002) The Adaptive Web: Adaptive Interfaces for Ubiquitous Web Access, Communications of the ACM, 45, 5, 34-38.

3. Burnkrant, R. E., and Unnava, H. R. (1989) Self-Referencing: A Strategy for Increasing Processing of Message Content, Personality and Social Psychology Bulletin, 15, 4, 628-638.

4. Burnkrant, R. E., and Unnava, H. R. (1995) Effects of Self-Referencing on Persuasion, Journal of Consumer Research, $22,1,17-26$.

5. Dasgupta, P., and Melliar-Smith, P. M. (2003) Dynamic Consumer Profiling and Tiered Pricing Using Software Agents, Electronic Commerce Research, 3, 3, 277-296.

6. Desai, K.K. and Hoyer, W.D. (2000) Descriptive Characteristics of Memory-Based Consideration Sets: Influence of Usage Occasion Frequency and Usage Location Familiarity, Journal of Consumer Research, 27, 3, 309-323.

7. Dhar, R. (1997) Consumer Preference for a No-Choice Option, Journal of Consumer Research, 24, 2, $215-231$.

8. Eirinaki, M., and Vazirgiannis, M. (2003) Web Mining for Web Personalization, ACM Transactions on Internet Technology, 3, 1, 1-27.

9. Foltz, P. W., and Dumais, S. T. (1992) Personalized Information Delivery: An Analysis of Information Filtering Methods, Communications of the ACM, 35, 12, 51-60.

10. Greenleaf, E.A. and Lehmann, D.R. (1995) Reasons for Substantial Delay in Consumer Decision Making, Journal of Consumer Research, 22, 2, 186-199.

11. Hauser, J.R. and Wernerfelt, B. (1989) The Competitive Implications of Relevant-Set/Response Analysis, Journal of Marketing Research, 26, 4, 391-405.

12. Howard, J.A., and Jadish N. Sheth, (1969) The Theory of Buyer Behavior, New York, NY: Wiley.

13. Howard, J.A. (1989) Consumer Behavior in Marketing Strategy. Englewood Cliffs, NJ: Prentice-Hall.

14. Huffman, C. and Kahn, B.E. (1998) Variety for Sale: Mass Customization or Mass Confusion? Journal of Retailing, 74, 4, 491-513.

15. Iyengar, S.S. and Lepper, M.R. (2000) When Choice is Demotivating: Can One Desire Too Much of a Good Thing? Journal of Personality and Social Psychology, 79, 6, 995-1006.

16. Johnson, M.D. (1984) Consumer Choice Strategies for Comparing Noncomparable Alternatives, Journal of Consumer Research, 11, 3, 741-753.

17. Johnson, M.D. (1986) Modeling Choice Strategies for Noncomparable Alternatives, Marketing Science, 5, 1, 37-54.

18. Kobsa, A. (2002) The Adaptive Web: Personalized Hypermedia and International Privacy, Communications of the ACM, $45,5,64-67$.

19. Ledford, J.a.L. (2002), Content Personalization Market Trends, Faulkner Information Services.

20. Levin, I.P. and Jasper, J.D. (1995) Phased Narrowing: A New Process Tracing Method for Decision Making, Organizational Behavior and Human Decision Processes, 64, 1, 1-8. 
21. Light, M., and Maybury, M. T. (2002) The Adaptive Web: Personalized Multimedia Information Access, Communications of the ACM, 45, 5, 54-59.

22. Loeb, S. (1992) Architecting Personalized Delivery of Multimedia Information, Communications of the ACM, 35, 12, $39-47$.

23. Maes, P., Guttman, R. H., and Moukas, A. G. (1999) Agents that Buy and Sell, Communications of the ACM, 42, 3, 8191.

24. Manber, U., Patel, A., and Robison, J. (2000) Experience with Personalization on Yahoo!, Communications of the ACM, $43,8,35-39$.

25. Moe, W. W. (2003) Buying, Searching, or Browsing: Differentiating Between Online Shoppers Using In-Store Navigational Clickstream, Journal of Consumer Psychology, 13, 1, 29-39.

26. Murthi, B.P.S., and Sarkar, S. (2003), "The Role of the Management Sciences in Research on Personalization," Management Science (49:10), 1344-1362.

27. Nedungadi, P. (1997) Recall and Consumer Consideration Sets Influencing Choice without Altering Brand Evaluations, Journal of Consumer Research, 23, 4, 263-277.

28. Peppers, D. and Rogers, M. (1997) Enterprise One to One: Tools for Competing in the Interactive Age. Doubleday, New York.

29. Perkowitz, M., and Etzioni, O. (2000) Adaptive Web Sites, Communications of the ACM, 43, 8, 152-158.

30. Pitkow, J., and Schütze, H., Cass, T., Cooley, R., Turnbull, D., Edmonds, A., Adar, E., and Breuel, T. (2002) The Consumer Side of Search: Personalized Search, Communications of the ACM, 45, 9, 50-55.

31. Porter, M. E. (2001) Strategy and the Internet. Harvard Business School Press.

32. Punj, G. and Brookes, R. (2001) Decision Constraints and Consideration Set Formation in Consumer Durables, Psychology and Marketing, 18, 8, 843-863.

33. Ramakrishnan, N. (2000) Pipe: Web Personalization by Partial Evaluation, IEEE Internet Computing, 4, 6, 21-31.

34. Roberts, J. and Lattin, J.M. (1991) Development and Testing of a Model of Consideration Set Composition, Journal of Marketing Research, 28, 4, 429-440.

35. Shahabi, C., and Banaei-Kashani, F. (2003) Efficient and Anonymous Web-Usage Mining for Web Personalization, Journal of Computing, 15, 2, 123-147.

36. Shugan, S.M. (1980) The Cost of Thinking, Journal of Consumer Research, 7, 2, 99-111.

37. Stewart, K. A., and Segars, A. H. (2002) An Empirical Examination of the Concern for Information Privacy Instrument, Information Systems Research, 13, 1, 36-49.

38. Tversky, A. (1977) Features of Similarity, Psychological Review, 84, 3, 327-352.

39. Volokh, E. (2000) Personalization and Privacy, Communications of the ACM, 43, 8, 84-85. 